Reprod. Nutr. Dévelop., 1988, 28 (5), 1317-1328

\title{
Acétylcarnitine et spermatozoïdes : relation avec la maturation épididymaire et la mobilité chez le verrat et I'homme
}

\author{
Claudette JEULIN $\left({ }^{1}\right)$, J. C. SOUFIR $\left({ }^{2}\right)$, Jocelyne MARSON $\left({ }^{1}\right)$, M. PAQUI- \\ GNON $\left({ }^{3}\right)$, J. L. DACHEUX $\left({ }^{3}\right)$ \\ (1) Laboratoire d'Histologie, $\left({ }^{2}\right)$ Laboratoire de Biologie Cellulaire, \\ C.H.U. Kremlin-Bicêtre, 94275 Kremlin-Bicêtre Cedex, \\ $\left({ }^{3}\right)$ Station de Physiologie de la Reproduction I.N.R.A., 37380 Monnaie, \\ France.
}

Summary. Acetylcarnitine and spermatozoa : relationship with epididymal maturation and motility in the boar and man.

The level of carnitine and acetylcarnitine in spermatozoa of boar epididymal origin and of human ejaculates was demonstrated. In the epididymal fluid of boars, the concentration of carnitine ( $\mathrm{nmol} / \mathrm{mg}$ protein) began to increase from 20 in the distal caput to rise progressively to 700 in the distal cauda. By contrast, the carnitine content of spermatozoa only started to increase in the proximal cauda where the concentration of carnitine in the fluid was $200-300 \mathrm{nmol} / \mathrm{mg}$ protein, then gradually increased in spermatozoa from more distal sites. The increase in the acetylcarnitine content of spermatozoa paralleled that of the carnitine amount, represented $50 \%$ of total carnitine (carnitine + acetylcarnitine) and coincided with the acquisition of progressive motility. In two populations of human seminal spermatozoa selected by migration and characterised by a very large difference in their percentage of progressively motile cells, higher carnitine and acetylcarnitine contents $(40 \%)$ were found in migrated spermatozoa compared to the residual population. These results suggest that accumulation of carnitine and its metabolite may be an important factor in the acquisition and the maintenance of progressive motility. Measurement of acetylcarnitine content of human seminal spermatozoa could be used as a marker of epididymal maturation.

\section{Introduction.}

L'épididyme est l'un des organes où la concentration de carnitine est la plus élevée (Marquis et Fritz, 1965b ; Casillas, 1972; Brooks, 1980). Elle est captée par l'épithélium épididymaire, s'accumule dans le fluide et pénètre dans les spermatozoïdes de différentes espèces au cours de leur maturation (Casillas, 1972, 1973 ; Casillas et Chaipayungpan, 1979 ; Inskeep et Hammerstedt, 1982 ; Casillas et al., 1984). In vitro, les spermatozoïdes épididymaires de taureau incubés en présence de substrats sont capables d'acétyler très rapidement la carnitine pour l'essentiel sous forme d'acétylcarnitine (Casillas et Erickson, 1975). 
In vitro, l'acétylcarnitine peut être métabolisée par les spermatozoïdes épididymaires de taureau lorsque les besoins en énergie deviennent très importants (Milkowski et al., 1976). L'évolution du gradient de concentration de carnitine au cours du transit épididymaire est variable selon les espèces. Chez le rat, la concentration de carnitine du plasma épididymaire croît à partir de la région distale de la tête épididymaire, de la région distale du corps chez le verrat et le bélier (Hinton et al., 1979) et du niveau proximal de la queue chez le lapin et le hamster (Hinton et al., 1979 ; Casillas et Chaipayungpan, 1979; Casillas et al., 1984). Les données en ce qui concerne les taux d'acétylcarnitine dans le plasma épididymaire et les spermatozoïdes sont contradictoires. Dans le fluide, la concentration augmenterait entre le corps et la queue de 10 fois chez le lapin et de 50 fois chez le hamster (Casillas et Chaipayungpan, 1979 ; Casillas et al., 1984). L'acétylcarnitine du plasma séminal humain n'est pas d'origine épididymaire et n'est pas un produit du métabolisme des spermatozoïdes (Soufir et Jeulin, 1985). Dans les spermatozoïdes la concentration d'acétylcarnitine resterait stable au cours du transit chez le hamster (Casillas et al., 1984), alors qu'elle s'accroît de 13 à $320 \mu \mathrm{mol} / 10^{8}$ spermatozoïdes entre la tête et la queue épididymaire chez le bélier (Inskeep et Hammerstedt, 1982). Le pourcentage de carnitine acétylée rapporté à la teneur totale en carnitine des spermatozoïdes est de 30 chez le hamster (Casillas et al., 1984), 80 chez l'homme (Brooks, 1979) et 85 chez le bélier (Inskeep et Hammerstedt, 1982).

Des spermatozoïdes immobiles, prélevés au niveau de la tête épididymaire de rat, initient une mobilité pendant de courtes périodes 2 à $20 \mathrm{~min}$ en présence de L-carnitine ou de L-acétylcarnitine (Hinton et al., 1981). L'acétylcarnitine augmente in vitro le pourcentage de spermatozoïdes mobiles d'éjaculats humains (Tanphaichitr, 1977 ; Jeulin et al., 1981). Chez I'homme la teneur en acétylcarnitine des spermatozoïdes est corrélée avec leur mobilité (Johansen et Bohmer, 1979) et le rapport acétylcarnitine sur carnitine des spermatozoïdes mobiles progressifs serait plus élevé que celui des vivants immobiles (Golan et al., 1984).

Ainsi l'analyse de la littérature fait apparaître des données variables, incomplètes et parfois contradictoires en particulier sur les points suivants: à quel niveau de l'épididyme la carnitine pénètre-t-elle dans les spermatozoïdes? Son entrée dans le fluide est-elle accompagnée immédiatement d'une élévation de la carnitine et de l'acétylcarnitine dans les spermatozoïdes et d'une modification de leur mobilité ? La mobilité des spermatozoïdes et la teneur en acétylcarnitine sont-elles liées?

C'est dans ce but que : 1) chez le verrat, nous avons étudié la distribution de la carnitine et de l'acétylcarnitine dans le plasma et les spermatozoïdes prélevés à différents niveaux de l'épididyme et 2) dans les spermatozoïdes humains, nous avons recherché si les spermatozoïdes mobiles progressifs étaient ceux dont la teneur en carnitine et en acétylcarnitine était la plus élevée.

\section{Matériel et méthodes.}

Préparation des échantillons de fluide épididymaire et de rete testis.

Le fluide épididymaire est collecté par microponction (Dacheux, 1980) dans 9 régions de 6 épididymes de verrat Large White, âgés de 180 jours. Chaque 
région (fig. 1) est perfusée avec $0,2 \mathrm{ml}$ d'une solution de Krebs-Ringer-bicarbonate (KRB) (Imai et al., 1979) saturée avec $5 \% \mathrm{CO}_{2}$ dans l'oxygène $(\mathrm{pH} 7,4)$ exceptée la région 9 , pour laquelle la perfusion est faite avec de l'huile de paraffine. Les échantillons sont centrifugés $(10000 \mathrm{~g}, 10 \mathrm{~min})$ et les surnageants (plasmas) conservés à $-20^{\circ} \mathrm{C}$. Le fluide du rete testis de 2 verrats est obtenu par cathétérisation (Edwards et al., 1976). Les plasmas sont préparés et conservés comme ci-dessus.

\section{Préparation des extraits de spermatozoïdes.}

Chez le verrat. - Les spermatozoïdes sont prélevés à partir de petites incisions dans le canal au niveau de la tête (régions 1 à 4 ) et de la queue (régions 8 à 9) de 6 épididymes (tabl. 1). Deux épididymes parmi les 6 , sont incisés au niveau de 9 régions (fig. 1 ). Les spermatozoïdes sont mis en suspensions dans $0,5 \mathrm{ml}$ de KRB, centrifugés $(1500 \mathrm{~g}, 15 \mathrm{~min}$ ) et déposés sur $4 \mathrm{ml}$ de Percoll à $30 \%$ dans la solution de KRB. Les culots de spermatozoïdes sont dilués dans $0,5 \mathrm{ml}$ de Triton $X 100$ (Sigma) à $0,2 \%$ dans le KRB, afin d'extraire la carnitine et l'acétylcarnitine par perméabilisation des membranes selon la méthode décrite par Johansen et Bohmer (1979). Les échantillons sont conservés à $-20^{\circ} \mathrm{C}$. La concentration des spermatozoïdes est mesurée à l'hémocytomètre.

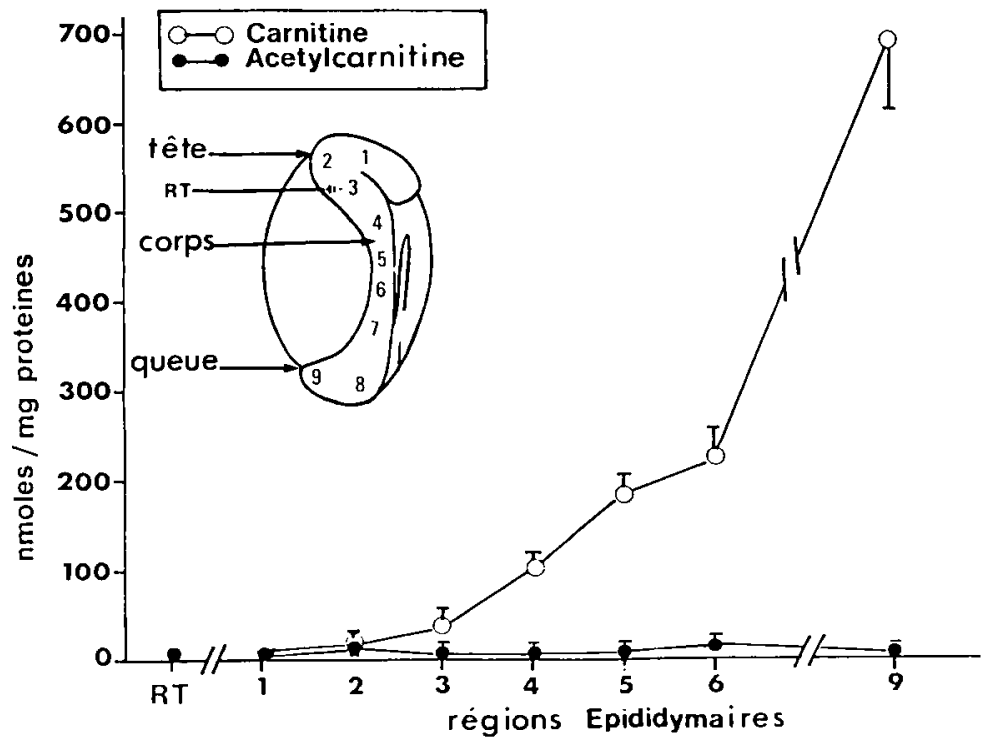

FIG. 1. - Concentrations de L. carnitine et $L$. acéty/carnitine dans le plasma du rete testis (RT) et dans 7 fractions provenant de 9 régions de plasmas épididymaires (voir schéma). Chaque point représente la moyenne \pm écart type rapporté à la moyenne de deux valeurs pour le plasma du rete testis et de 6 valeurs pour les échantillons épididymaires. 
TABLEAU 1

Teneurs en L. carnitine et $L$. acéty/carnitine des spermatozoïdes de verrat ( $\mathrm{nmol} / 10^{8}$ spermatozoïdes) prélevés au niveau de la tête et de la queue de l'épididyme.

\begin{tabular}{|c|c|c|c|}
\hline Région & Carnitine & Acétylcarnitine & Acétylation (\%) \\
\hline $\begin{array}{l}\text { Tête (régions } 1 \text { à } 4 \text { ) } \\
\text { Queue (régions } 8 \text { et } 9 \text { ) }\end{array}$ & $\begin{array}{r}7,4 \pm 1,4 \\
51,4 \pm 4,0^{*}\end{array}$ & $\begin{array}{l}12,2 \pm 3,2 \\
57,0 \pm 13,0\end{array}$ & $\begin{array}{l}62 \\
52,5\end{array}$ \\
\hline
\end{tabular}

Moyenne \pm écart type rapporté à la moyenne, obtenue avec 6 épididymes.

* $p<0,01$, comparaison avec les valeurs obtenues pour la tête (test non paramétrique de Kruskall-Wallis).

Chez l'homme. - Un groupe de 15 donneurs (étudiants en médecine) a été sélectionné en fonction de la normalité des caractéristiques de leur sperme (concentration $>37 \cdot 10^{6} / \mathrm{ml}$, mobilité $>50 \%$, vitalité > $63 \%$, morphologie $>51 \%$ normaux, abstinence sexuelle de 3 à 5 jours). Deux populations de spermatozoïdes caractérisées par une proportion très différentes de mobiles progressifs est obtenue par migration descendante dans une solution d'albumine selon la méthode décrite par Ericsson et al. (1973). Une demi-heure après émission, les éjaculats sont dilués volume à volume dans une solution de Tyrode $(\mathrm{pH} 7,5)$. Les échantillons sont centrifugés $(600 \mathrm{~g}, 10 \mathrm{~min})$ et la concentration des spermatozoïdes remis en suspension à partir du culot est ajustée entre 5 et $10 \cdot 10^{7} / \mathrm{ml}$. Deux à 6 colonnes de verre contenant chacune $1,8 \mathrm{ml}$ de sérum albumine bovine à $10 \%$ dans le Tyrode sont préparées et $1 \mathrm{ml}$ de la suspension de spermatozoïdes lavés est déposé sur chacune d'entre elles. Après $1 \mathrm{~h} 30$ d'incubation à température ambiante, les fractions supérieures (environ $1,2 \mathrm{ml} \times$ nombre de colonnes) sont prélevées et constituent la population « résiduelle » de spermatozoïdes n'ayant pas migré. Les fractions inférieures $(1,6 \mathrm{ml} \times$ nombre de colonnes) sont collectées, diluées dans le Tyrode $(1: 10)$, centrifugées (600 g. $10 \mathrm{~min}$ ) et remises en suspension dans $1 \mathrm{ml}$ de Tyrode. Cette population est constituée de spermatozoïdes « sélectionnés » par migration dans l'albumine. Les concentrations sont mesurées et la perméabilisation des membranes des deux populations de spermatozoïdes est obtenue par le Triton X 100 à $1 \%$ dans l'eau distillée $\left(0,250 \mathrm{ml}\right.$ pour $50 \cdot 10^{6} \mathrm{sp}$.).

Analyse de la mobilité spermatique.

La mobilité des spermatozoïdes humains et de verrat est analysée par microscopie à contraste de phase entre lame et lamelle (épaisseur de la suspension $28 \mu \mathrm{m}$ ). Chaque spermatozoïde est classé en immobile, mobile non progressif et mobile progressif. La seconde catégorie correspond à des mouvements désorientés du flagelle et/ou de la tête.

Analyse de la vitalité.

Elle est évaluée uniquement pour les échantillons de sperme humain par comptage des spermatozoïdes colorés par l'éosine et la nigrosine (Sigma Chemical Co., St Louis, MO, USA) (Eliasson, 1977). 
Dosages biochimiques.

La déprotéinisation des échantillons de plasmas épididymaires et de rete testis est effectuée par ébullition pendant $3 \mathrm{~min}$ et centrifugation $14000 \mathrm{~g}$, $10 \mathrm{~min}$. Celle des extraits de spermatozoïdes humains et de verrat par centrifugation sur cône de filtration moléculaire (C F 50 A, Amicon), (1000 g, $20 \mathrm{~min}$, $\left.+4{ }^{\circ} \mathrm{C}\right)$. Les dosages de la $\mathrm{L}(-)$ carnitine libre dans les échantillons de plasmas et les extraits de spermatozoïdes sont effectués en spectrophotométrie (technique décrite par Marquis et Fritz, 1964, modifiée par Soufir et al., 1981). L'acétylcarnitine est dosée également par spectrophotométrie [technique de Marquis et Fritz (1965a) et Pearson et al. (1974)]. Comme le plasma épididymaire a été dilué par la solution de perfusion, nous avons mesuré la concentration de protéines (Lowry et al., 1951) de chaque échantillon afin d'exprimer les résultats par $\mathrm{mg}$ de protéines dans la figure 1.

\section{Résultats.}

Distribution de la carnitine et de l'acéty/carnitine dans le plasma et les spermatozoïdes épididymaires de verrat.

La figure 1 représente les distributions de carnitine libre et d'acétylcarnitine dans le plasma du rete testis et des différentes régions de l'épididyme. La concentration de carnitine est faible au niveau du rete testis et dans le plasma de la tête proximale $(16 \mathrm{nmol} / \mathrm{mg}$ de protéines). Elle s'accroît au niveau de la région distale de la tête $(20 \mathrm{nmol} / \mathrm{mg}$ de protéines, région 3) pour atteindre $200 \mathrm{nmol} / \mathrm{mg}$ de protéines région 5 . Les valeurs maximales sont observées au niveau de la partie caudale $(700 \mathrm{nmol} / \mathrm{mg}$ de protéines ou $14,5 \pm 1,2 \mu \mathrm{mol} / \mathrm{ml}$ ). Au contraire, la concentration d'acétylcarnitine reste faible $(7-15 \mathrm{nmol} / \mathrm{ml}$ de protéine) tout au long de l'épididyme.

Les teneurs en carnitine et en acétylcarnitine des spermatozoïdes prélevés au niveau de la tête et de la queue de l'épididyme sont indiquées dans le tableau 1. Le taux des 2 composés augmente au cours du transit (fig. 2). De la région 2 à la région 6 , la teneur en carnitine et en acétylcarnitine des spermatozoïdes est faible (environ $7-10 \mathrm{nmol} / 10^{8}$ spermatozoïdes). Un accroissement d'au moins 5 fois la valeur initiale survient à partir de la région 7 jusqu'à la partie distale de la queue épididymaire. Le rapport acétylcarnitine/carnitine est constant pendant toute la durée du transit. L'augmentation de la teneur en acétylcarnitine coïncide avec celle de la carnitine et correspond à $50 \%$ de la teneur en carnitine totale (carnitine + acétylcarnitine) (tabl. 1).

L'évolution de la mobilité des spermatozoïdes au cours du transit est représentée dans la figure 3 . Le pourcentage de spermatozoïdes mobiles (progressifs et non progressifs) augmente seulement lorsque les gamètes atteignent la région 3 , et croît régulièrement jusqu'à la région caudale. Par contre le pourcentage de spermatozoïdes mobiles progressifs reste faible de la région 2 à la région 6 , puis augmente brusquement de la région 6 à la partie terminale de la queue de l'épididyme. 


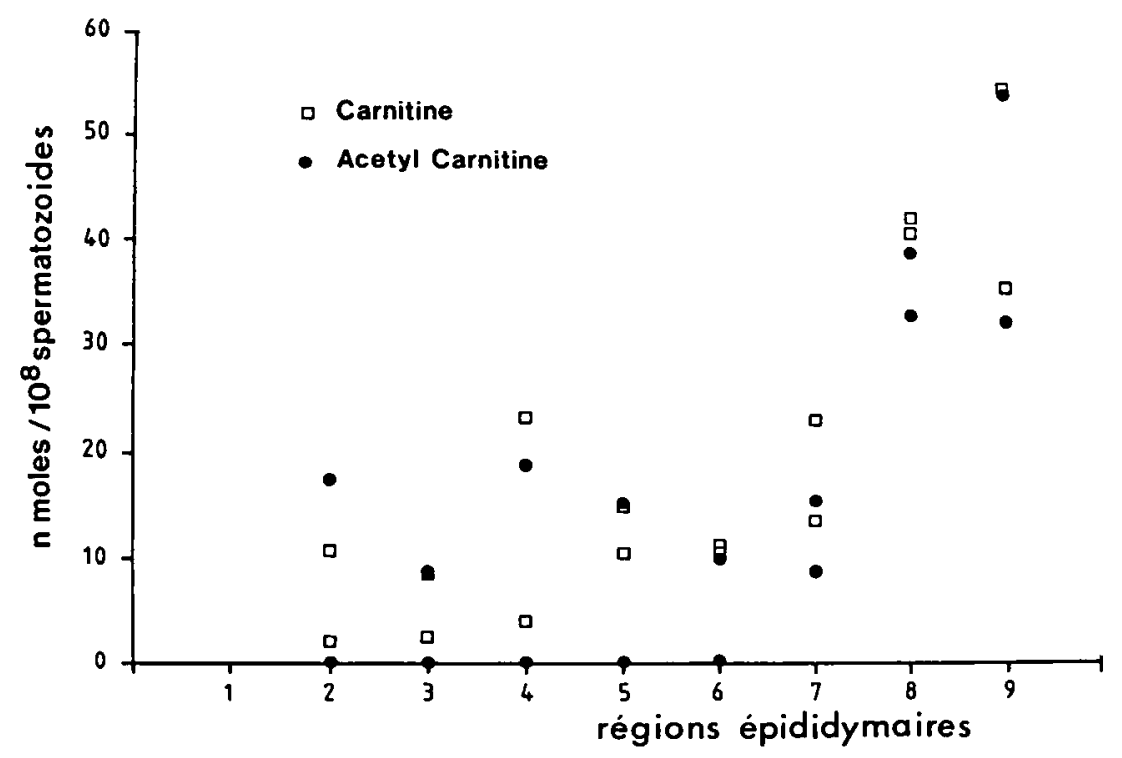

FIG. 2. - Teneurs en L. carnitine et en L. acéty/carnitine des spermatozoides prélevés dans 9 régions de 2 épididymes.

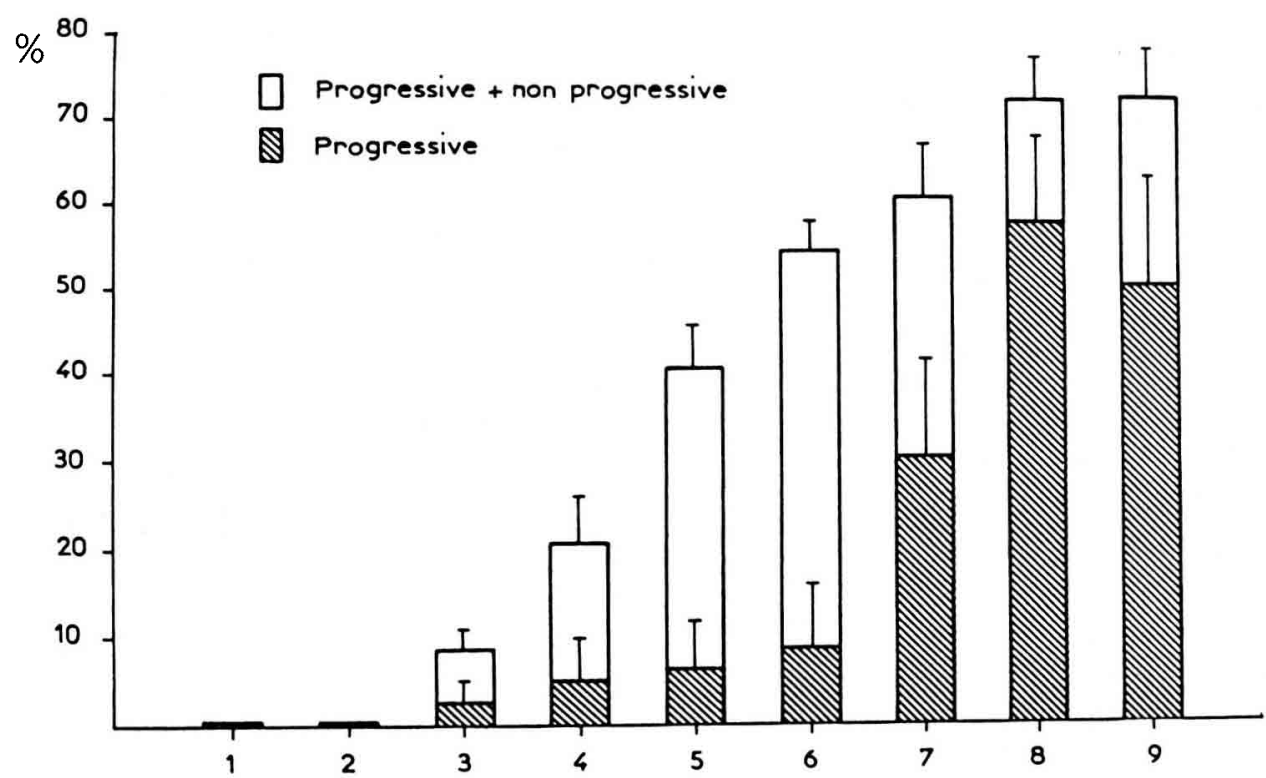

FIG. 3. - Pourcentage de spermatozoïdes mobiles observés au microscope à contraste de phase dans 9 régions épididymaires. Chaque point représente la moyenne \pm écart type rapporté à la moyenne de 8 valeurs. 
Relation entre carnitine, acéty/carnitine et mobilité des spermatozoïdes humains.

Les caractéristiques des deux populations de spermatozoïdes obtenues par migration sont indiquées dans le tableau 2. Le pourcentage moyen de spermatozoïdes mobiles (progressifs et non progressifs) est très différent entre les populations résiduelle ( $26 \%$ ) et sélectionnée ( $89 \%$ ). Compte tenu de la sélection des éjaculats, la différence des pourcentages moyens de vitalité entre les deux populations est moins importante $81 / 100 \%$. Les teneurs en carnitine et en acétylcarnitine ( $\mathrm{nmol} / 10^{8}$ spermatozoïdes vivants) sont en moyenne significativement plus élevées dans la population de spermatozoïdes sélectionnées par migration comparées à celles mesurées dans la population résiduelle ne migrant pas (carnitine 51/37, acétylcarnitine 130/93). La teneur en carnitine totale (carnitine + acétylcarnitine) est plus élevée dans la population sélectionnée $181 / 129,5$ dans la population résiduelle. Le pourcentage de carnitine acétylée est identique dans les deux populations (72\%).

TABLEAU 2

Carnitine, acéty/carnitine et caractéristiques des populations de spermatozö̈des humains isolées par migration.

\begin{tabular}{|c|c|c|c|c|c|c|c|c|}
\hline \multirow{4}{*}{ Echantillons } & \multicolumn{8}{|c|}{ Populations de spermatozoïdes } \\
\hline & \multicolumn{4}{|c|}{ Résiduelle } & \multicolumn{4}{|c|}{ Sélectionnée } \\
\hline & \multirow{2}{*}{$\begin{array}{c}\text { Mobilité } \\
(\%)\end{array}$} & \multirow{2}{*}{$\begin{array}{c}\text { Vitalité } \\
(\%)\end{array}$} & Carnitine & Acétylcarnitine & \multirow{2}{*}{$\begin{array}{l}\text { Mobilité } \\
\text { ts) }(\%)\end{array}$} & \multirow{2}{*}{$\begin{array}{c}\text { Vitalité } \\
(\%)\end{array}$} & \multicolumn{2}{|c|}{ Carnitine Acétylcarnitine } \\
\hline & & & \multicolumn{2}{|c|}{ (nmoles $/ 10^{8}$ sperm. vivants) } & & & \multicolumn{2}{|c|}{ (nmoles $/ 10^{8}$ sperm. vivants } \\
\hline 1 & 25 & 63 & 27 & 68 & 90 & 100 & 61 & 202 \\
\hline 2 & 50 & 80 & 62 & 129 & 95 & 100 & 60 & 146 \\
\hline 3 & 30 & 85 & 55 & 83 & 90 & 100 & 41 & 103 \\
\hline 4 & 25 & 80 & 38 & 67 & 80 & 100 & 61 & 122 \\
\hline 5 & 10 & 71 & 59 & 104 & 90 & 100 & 26 & 70 \\
\hline 6 & 20 & 89 & 22 & 71 & 95 & 100 & 27 & 47 \\
\hline 7 & 30 & 89 & 18 & 37 & 90 & 100 & 46 & 85 \\
\hline 8 & 10 & 80 & 18 & 129 & 80 & 100 & 14 & 76 \\
\hline 9 & 30 & 95 & 38 & 77 & 90 & 100 & 74 & 100 \\
\hline 10 & 5 & 75 & 29 & 38 & 80 & 100 & 99 & 107 \\
\hline 11 & 35 & 80 & 31 & 128 & 80 & 100 & 18 & 120 \\
\hline 12 & 15 & 85 & 19 & 62 & 100 & 100 & 68 & 106 \\
\hline 13 & 15 & 79 & 39 & 130 & 100 & 100 & 64 & 288 \\
\hline 14 & 50 & 95 & 39 & 159 & 90 & 100 & 35 & 176 \\
\hline 15 & 45 & 75 & 54 & 118 & 90 & 100 & 67 & 196 \\
\hline 15 & $\begin{array}{c}26 \\
\pm 14\end{array}$ & $\begin{array}{c}81 \\
\pm 9\end{array}$ & $\begin{array}{c}36,5 a \\
+15\end{array}$ & $\begin{array}{l}93 \mathrm{~b} \\
\pm 37\end{array}$ & $\begin{array}{l}89 \\
\pm \quad 7\end{array}$ & 100 & $\begin{array}{c}51 a \\
24\end{array}$ & $\begin{array}{r}130 \mathrm{~b} \\
\pm 63\end{array}$ \\
\hline
\end{tabular}

Différences significatives entre les deux populations résiduelle et sélectionnée : a : $0,005<p<$ 0,$01 ; b: p<0,01$ par le test non paramétrique $T$ de Wilcoxon (séries appariées). 


\section{Discussion.}

Nos résultats confirment les observations précédentes montrant que la carnitine s'accumule dans le fluide puis dans les spermatozoïdes épididymaires au cours de leur transit (Hinton et al., 1979; Casillas, 1973; Casillas et Chaipayungpan, 1979 ; Casillas et al., 1984). Ils précisent qu'il existe un délai entre l'élévation du taux de carnitine dans le plasma et l'augmentation de la charge en carnitine des spermatozoïdes épididymaires de verrat. La concentration de carnitine dans le plasma s'élève au niveau de la partie distale de la tête (régions 3-4) alors que la teneur en carnitine des spermatozoïdes ne s'accroît franchement que dans la région caudale (8-9). L'existence d'un délai entre l'accumulation de carnitine dans le plasma et son entrée dans les spermatozoïdes suggère que la membrane du spermatozoïde n'est pas facilement perméable à la carnitine (Casillas, 1973; Brooks, 1979). Si nous supposons que le volume aqueux du spermatozoïde est à peu près identique chez le verrat, chez l'homme (environ 2,5 $\mu \mathrm{l} / 10^{8}$ spermatozoïdes) (Ford et Harrison, 1983) et chez le bélier (Hammerstedt et al., 1979), nous pouvons calculer la concentration intracellulaire de carnitine des spermatozoïdes de la queue. Elle est de l'ordre de 20-25 $\mathrm{mol} / \mathrm{ml}$, donc très proche de la concentration de carnitine dans le plasma environnant $(14,5 \mu \mathrm{mol} / \mathrm{ml})$. Ainsi la concentration de carnitine est sensiblement la même à l'intérieur et à l'extérieur des spermatozoïdes. Ceci suggère une diffusion passive de la carnitine dans la cellule puis la création d'un équilibre physique entre milieu intra- et extra-cellulaire. Toutefois, à partir d'un certain seuil le compartiment intracellulaire est saturé et des concentrations élevées de carnitine exogène ne peuvent plus le modifier (Casillas, 1973). Le fait qu'il faille un certain taux de carnitine dans le plasma environnant $(300 \mu \mathrm{mol} / \mathrm{mg}$ protéines $)$ pour qu'il y ait pénétration dans le spermatozoïde évoque deux possibilités : soit cette molécule en partie hydrophile passe difficilement la membrane plasmique, soit des modifications structurales de la membrane plasmique faciliteraient une diffusion passive de la carnitine à partir des niveaux 6-7. Quel que soit ce mécanisme, il s'accompagne d'un taux de captation de carnitine par le spermatozoïde très faible (Casillas, 1973) et un équilibre entre milieu intra- et extra-cellulaire ne peut être atteint qu'après plusieurs heures de transit dans le corps.

De façon concomitante à la pénétration de carnitine dans la cellule, il se produit une synthèse d'acétylcarnitine qui ne s'accompagne pas de la libération de ce composé dans le milieu extra-cellulaire. Le pourcentage de carnitine acétylée dans les spermatozoïdes épididymaires de verrat est compris entre 50 et $60 \%$. Il apparaît plus faible que celui des spermatozoïdes humains éjaculés [87 \% pour Brooks (1979); $72 \%$ dans cette étude]. Quel que soit le niveau de l'épididyme le rapport acétylcarnitine/carnitine totale n'est pas modifié. Ceci confirme bien que l'acétylcarnitine transférase présente dès le stade spermatocyte primaire diplotène (Vernon et al., 1971) est fonctionnelle.

Au cours du transit épididymaire, des modifications de la mobilité des spermatozoïdes de rat ont été associées à l'accumulation de carnitine dans les spermatozoïdes (Hinton et al., 1981). Par contre chez le verrat, nous montrons que l'apparition de cellules mobiles dans la région de la tête épididymaire ne s'accompagne pas d'une pénétration de carnitine dans les spermatozoïdes 
puisque cette dernière ne survient qu'au niveau distal. La pénétration de carnitine et sa transformation en acétylcarnitine s'accompagnent d'une modification du type de mouvement (mobilité progressive). Ces observations suggèrent que la carnitine ne serait pas impliquée dans l'initiation de la mobilité mais jouerait un rôle dans l'acquisition d'un mouvement orienté et coordonné. Cette relation entre mobilité progressive et charge en carnitine des spermatozoïdes est développée dans notre étude chez l'homme. L'isolement par migration de deux populations présentant des pourcentages de spermatozoïdes mobiles progressifs très différents 26 et $89 \%$, montre que des cellules sélectionnées ont une teneur moyenne plus élevée (40\%) en carnitine et en acétylcarnitine que les spermatozoïdes résiduels ne migrant pas (tabl. 2). Des différences du même ordre ont été obtenues par Golan et al. (1984) sur quelques échantillons de spermatozoïdes humains isolés par migration dans leur propre plasma séminal.

Différentes hypothèses peuvent expliquer le rôle de l'acétylcarnitine dans l'acquisition et le maintien d'une mobilité progressive. L'acétylcarnitine constitue un pool énergétique immédiatement mobilisable (Milkowski et al., 1976), produisant l'énergie nécessaire au mouvement progressif. Cette voie pourrait être plus importante que d'autres processus métaboliques (glycolyse ou $\beta$-oxydation). D'autre part, la population résiduelle moins progressive, pourrait refléter une certaine immaturité pour des raisons variées (durée de transit trop rapide, immaturité membranaire ne permettant pas la pénétration de carnitine, etc...). Ces résultats suggèrent que la charge en carnitine et surtout en acétylcarnitine des spermatozoïdes pourrait être considérée comme des marqueurs de la qualité de la maturation. Une des applications cliniques de ces résultats consisterait à mesurer la teneur en acétylcarnitine des spermatozoïdes éjaculés humains afin de déterminer leur degré de maturation épididymaire.

$5^{e}$ Congrès de la Société d'Andrologie de langue française, Paris, décembre 1987.

Remerciements. - Cette recherche a bénéficié d'une aide de l'Institut National de la Recherche Médicale (INSERM) contrat $N^{\circ} 135.007$. Une partie de ces résultats (verrat) a déjà été publié (J. Reprod. Fertil., 1987, 79, 523-529).

\section{References}

BROOKS D. E., 1979. Carnitine acetylcarnitine and the activity of carnitine acetyltransferases in seminal plasma and spermatozoa of men, rams and rats. J. Reprod. Fert., 56, 667-673.

BROOKS D. E., 1980. Carnitine in the male reproductive tract and its relation to the metabolism of the epididymis and spermatozoa, 219-235. In FRENKEL R. A., MAC GARRY J. D., Carnitine biosynthesis metabolism and functions, Acad. Press, New York.

BIOCHEMICA INFORMATION I., 1973. Boehringer, Mannheim, GMBH 11.

CASILLAS E. R., 1972. The distribution of carnitine in male reproductive tissues and its effect on palmitate oxydation by spermatozoal particles. Biochim. Biophys. Acta, 280, 545-551.

CASILLAS E. R., 1973. Accumulation of carnitine by bovine spermatozoa during maturation in the epididymis. J. biol. Chem., 248, 8227-8232. 
CASILLAS E. R., ERICKSON B. J., 1975. The role of carnitine in spermatozoan metabolism: substrate-induced elevations in the acetylation state of carnitine and coenzyme-A in bovine and monkey spermatozoa. Biol. Reprod., 12, 275-283.

CASILLAS E. R., CHAIPAYUNGPAN S., 1979. The distribution of carnitine and acetylcarnitine in the rabbit epididymis and the carnitine content of rabbit spermatozoa during maturation. $J$. Reprod. Fert., 56, 439-444.

CASILLAS E. R., VILLALOBOS P., GONZALES R., 1984. Distribution of carnitine and acetylcarnitine in the hamster epididymis and in epididymal spermatozoa during maturation. J. Reprod. Fert., 72, 197-201.

DACHEUX J. L., 1980. An in vitro luminal perfusion technique to study epididymal secretion. IRCS med. Sci., 8, 137.

EDWARDS E. M., DACHEUX J. L., WAITES G. M. H., 1976. Effects of $\alpha$-chlorohydrin on the metabolism of testicular and epididymal spermatozoa of ram. J. Reprod. Fert., 48, 265-270

ELIASSON R., 1977. Semen analysis and laboratory workup, 169-188. In COCKETT A. T. K., URRY R. L., Male infertility, workup, treatment and research, Grune et Stratton, New York.

ERICSSON R. J., LANGEVIN C. N., NISHINO M., 1973. Isolation of fractions rich in human $Y$ sperm. Nature, Lond., 246, $421-424$.

FORD W. C. L., HARRISON A., 1983. D- $\left(1-{ }^{14} \mathrm{C}\right)$ mannitol and $\left(U-{ }^{14} \mathrm{C}\right)$ sucrose as extracellular space markers for human spermatozoa and the uptake of 2-deoxyglucose. $J$. Reprod. Fert. 69, 479-487.

GOLAN R., WAISSENBERG T. R., LEWIN L. M., 1984. Carnitine and acetylcarnitine in motile and immotile human spermatozoa. Int. J. Androl., 7, 484-494.

HAMMERSTEDT R. H., KEITZ A. D., HAY S., DELUCA N., AMANN R. P., 1979. Changes in ram sperm membranes during epididymal transit. Arch. Biochem. Biophys., 196, 7-12.

HINTON B. T., SNOSWELL A. M., SETCHELL B. P., 1979. The concentration of carnitine in the luminal fluid of the testis and epididymis of the rat and some other mammals. J. Reprod. Fert. $56,105-111$.

HINTON B. T., BROOKS D. E., DOTT H. M., SETCHELL B. P., 1981. Effects of carnitine and some related compounds on the motility of rat spermatozoa from the caput epididymis. J. Reprod. Fert., 61, 59-64.

IMAI H., NIWNA K., IRITANI A., 1979. Time requirement of capacitation of boar spermatozoa assessed by their ability to penetrate the zona-free hamster egg. $J$. Reprod. Fert., 56. 489-492.

INSKEEP P. B., HAMMERSTEDT R. H., 1982. Changes in metabolism of ram sperm associated with epididymal transit or induced by exogenous carnitine. Biol. Reprod., 27, 735-743.

JEULIN C., SOUFIR J. C., JOUANNET P., 1981. The effects of L. carnitine and D. L. acetylcarnitine on human sperm motility as measured by laser doppler velocimetry. I.R.C.S. med. Sci., 9. 722-723.

JOHANSEN L., BOHMER T., 1979. Motility related to the presence of carnitine/acetylcarnitine in human spermatozoa. Int. J. Androl., 2, 202-210.

LOWRY O. H., ROSEBOUGH M. T., FARR A. L., RANDALL P. J., 1951. Protein measurement with the Folin phenol reagent. J. biol. Chem., 193, 265-275.

MARQUIS N. R. P., FRITZ I. B., 1964. Enzymological determination of free carnitine concentrations in rat tissues. J. Lipid Res., 5, 184-187

MARQUIS N. R. P., FRITZ I. B., 1965a. The distribution of carnitine acetylcarnitine and carnitine acetyl transferase in rat tissues. J. biol. Chem., 240, 2193-2197.

MARQUIS N. R. P., FRITZ I. B., 196£ J. Effects of testosterone on the distribution of carnitine, acetylcarnitine and carnitine acetyltransferase in tissues of the reproductive system of the male rat. J. biol. Chem., 240, 2197-2200.

MILKOWSKI A. L., BABCOCK D. F., LARDY H. A., 1976. Activation of bovine epididymal sperm respiration by caffeine. Its transient nature and relationship to the utilization of acetylcarnitine. Arch. Biochem. Biophys., 176, 250-256.

PEARSON D. J., TUBBS P. K., CHASE F. A., 1974. Carnitine and acylcarnitines, 1758-1771. In BERGMEYER H. U., Methods of enzymatic analysis, Verlag Chemie Weinheim.

SOUFIR J. C., JEULIN C., 1985. Origin of L-acetyl carnitine of human seminal plasma. Prog. Reprod. Biol. Med., 12, 99-103. 
SOUFIR J. C., MARSON J., JOUANNET P., 1981. Free (L) carnitine in human seminal plasma. Int. J. Androl., 4, 388-393.

VERNON R. G., GO V. L. W., FRITZI. B., 1971. Studies on spermatogenesis in rats. Il. Evidence that acetyl carnitine transferase is a marker enzyme for the investigation of germ cell differentiation. Can. J. Biochem., 49, 761-767.

TANPHAICHITR N., 1977. In vitro stimulation of human sperm motility by acetylcarnitine. Int. J. Fertil., 22, 85-91. 